Article

\title{
Quantitative Vulnerability Assessment of Cyber Security for Distribution Automation Systems
}

\author{
Xiaming Ye ${ }^{1}$, Junhua Zhao ${ }^{2, *}$, Yan Zhang ${ }^{1}$ and Fushuan Wen ${ }^{3}$ \\ 1 College of Electrical Engineering, Zhejiang University, Hangzhou 310027, China; \\ E-Mails: yexiaming@zju.edu.cn (X.Y.); zhangyan_1990@126.com (Y.Z.) \\ 2 School of Electrical Engineering and Computer Science, the University of Newcastle, \\ Newcastle, NSW 2308, Australia \\ 3 Department of Electrical and Electronic Engineering, Institut Teknologi Brunei, \\ Bandar Seri Begawan BE1410, Brunei; E-Mail: fushuan.wen@gmail.com \\ * Author to whom correspondence should be addressed; E-Mail: fuxiharp@gmail.com; \\ Tel.: +86-571-8795-3133; Fax: +86-571-8795-2869.
}

Academic Editor: Thorsten Staake

Received: 19 March 2015 / Accepted: 25 May 2015 / Published: 3 June 2015

\begin{abstract}
The distribution automation system (DAS) is vulnerable to cyber-attacks due to the widespread use of terminal devices and standard communication protocols. On account of the cost of defense, it is impossible to ensure the security of every device in the DAS. Given this background, a novel quantitative vulnerability assessment model of cyber security for DAS is developed in this paper. In the assessment model, the potential physical consequences of cyber-attacks are analyzed from two levels: terminal device level and control center server level. Then, the attack process is modeled based on game theory and the relationships among different vulnerabilities are analyzed by introducing a vulnerability adjacency matrix. Finally, the application process of the proposed methodology is illustrated through a case study based on bus 2 of the Roy Billinton Test System (RBTS). The results demonstrate the reasonability and effectiveness of the proposed methodology.
\end{abstract}

Keywords: smart grid; distribution automation system; cyber security; vulnerability assessment 


\section{Introduction}

The seamless merging of traditional power systems with cutting-edge information technologies has become an inevitable trend in smart grids [1,2]. In a power distribution system, with the help of advanced information technologies and intelligent feeder remote terminal units (FRTU), a distribution automation system (DAS) is able to provide higher reliability, greater efficiency and intensive interactions with consumers [3].

Nonetheless, the adoption of common communication protocols and deployment of various intelligent electronic devices (IED) introduces more vulnerabilities which can be used by cyber attackers [4]. Moreover, the tighter integration of cyber systems and physical power systems can easily lead to cyber-attacks that can degrade control performance or even cause power outages in a smart grid $[5,6]$. Thus, knowing how to deal with the cyber security issues of smart grids has become a new challenge. Some basic guidelines for cyber security have been published [7,8] and some studies on the cyber security of power control systems have been carried out in the past few years [9-14]. The existence of exploitable vulnerabilities is the precondition for cyber-attacks.

However, most of the existing cyber security studies focus on the control systems in power plants or substations. In these studies, the intelligent terminal devices are usually located in restricted areas. On the contrary, the terminal devices in a DAS are usually located at remote areas with limited physical protection, e.g., FRTUs [15]. These terminal devices act as widespread real-time monitors and intelligent controllers in a distribution system, and can exchange measurement data and control commands with the DAS control center server through communication network in normal operations. As for cyber-attacks, an attacker can penetrate FRTUs or other terminal devices via the modems between them and the communication network. On account of the limited computational capacity, most of the effective security measures found in computer networks cannot be used directly in the terminal devices [16]. Moreover, the intelligent terminal devices in a DAS will support more open and standardized communication protocols such as IEC 61850 in the near future [17]. For these reasons, the DAS is more vulnerable to cyber-attacks and therefore the security issues in a DAS should be properly addressed.

It is worth noting that ensuring the complete security of every single device in the DAS is hardly possible from the point of view of the cost of implementing the security measures [18]. Thus, an assessment framework for vulnerability ranking in a DAS is urgently required. The common vulnerability scoring system (CVSS) provides an open framework for vulnerability assessment [19]. It evaluates the impacts of vulnerabilities in computer networks from three aspects: base, temporal and environmental. However, the CVSS scores each vulnerability independently. It cannot analyze the impacts of cyber- attacks on physical systems, and does not take the interactions among different vulnerabilities into consideration. Ten [11] proposed an assessment framework to evaluate the vulnerability of supervisory control and data acquisition (SCADA) systems. Zonous [13] presented a unified formalism to model cyber-physical systems and proposed a vulnerability ranking method according to the potential physical consequences as well as attack complexity. However, these researches mainly focus on the cyber-attacks against power transmission systems and the interaction between attackers and defenders has not been analysed.

This paper focuses on the vulnerability assessment issue in a DAS. The purposes are to study the potential physical consequences of cyber-attacks on a DAS and to help system operators rank the 
vulnerabilities so as to more effectively enhance the cyber security of a DAS. The main contribution of this paper is proposing an original vulnerability assessment model to rank the vulnerabilities in a DAS based on potential consequences of cyber-attacks and the relationship among different vulnerabilities. Specifically, the potential physical consequences of cyber-attacks are discussed from two aspects: terminal devices and control center servers. The attack processes are modeled as a series of attack-defense games (ADGs) and relationships among different vulnerabilities are analyzed by introducing vulnerability adjacency matrix.

The rest of this paper is organized as follows: Section 2 presents the overall process of vulnerability assessment. Section 3 analyzes the physical consequences of cyber-attacks. Section 4 and Section 5 build the game model and introduce the vulnerability adjacency matrix to analyze the relationship among vulnerabilities, respectively. A case study based on bus 2 of the Roy Billinton Test System (RBTS) is used to illustrate how to apply the proposed method in Section 6. Finally, some conclusions are given in Section 7.

\section{Outline of Methodology}

The assessment methodology can be divided into three parts: physical consequences analysis, attack processes modeling and vulnerability adjacency matrix formation.

\subsection{Physical Consequences}

Potential consequences of cyber-attacks include revealing utilities' and consumers' private information, economic losses, and loss of load [18]. This paper mainly focuses on the control performance of the DAS, so the physical consequences are assessed by the quantity of loss of load and its duration.

A successful cyber-attack will result in a violation of all or part of the security properties (the integrity, availability, and confidentiality) [20]. Loss of different security properties leads to different physical consequences. Loss of integrity renders attackers the ability to change the control commands or measurement data. For instance, an attacker can send modified control commands to trip the switches in a distribution system, or send incorrect measurement data to misguide the decision-making of control strategies, both of which will result in unnecessary loss of load. Loss of availability renders the operators unable to collect measurement data or control the terminal devices, but it would not lead to severe physical consequences directly and immediately [21]. For example, a denial of service (DoS) attack on a relay protection IED will not affect the normal operation of a distribution system until a fault occurs. Confidentiality is usually the main concern in terms of personal privacy [22] and the leakage of system settings can ultimately lead to integrity or availability attacks [23]. However, compared with integrity and availability, loss of confidentiality will not affect the control performance of the DAS directly in most cases [15]. Thus, the physical consequences of cyber-attacks on confidentiality are not the research emphasis of this paper.

A DAS consists of a control center, terminal devices and a communication network. The communication architecture of a DAS is depicted in Figure 1. Except for the remote monitoring and control under normal operations, the fault detection, isolation and restoration are the most important 
functions in a DAS which can enhance the reliability of a distribution system. There are primarily two restoration schemes in distribution systems [24,25], both of which will be discussed in Section 3.

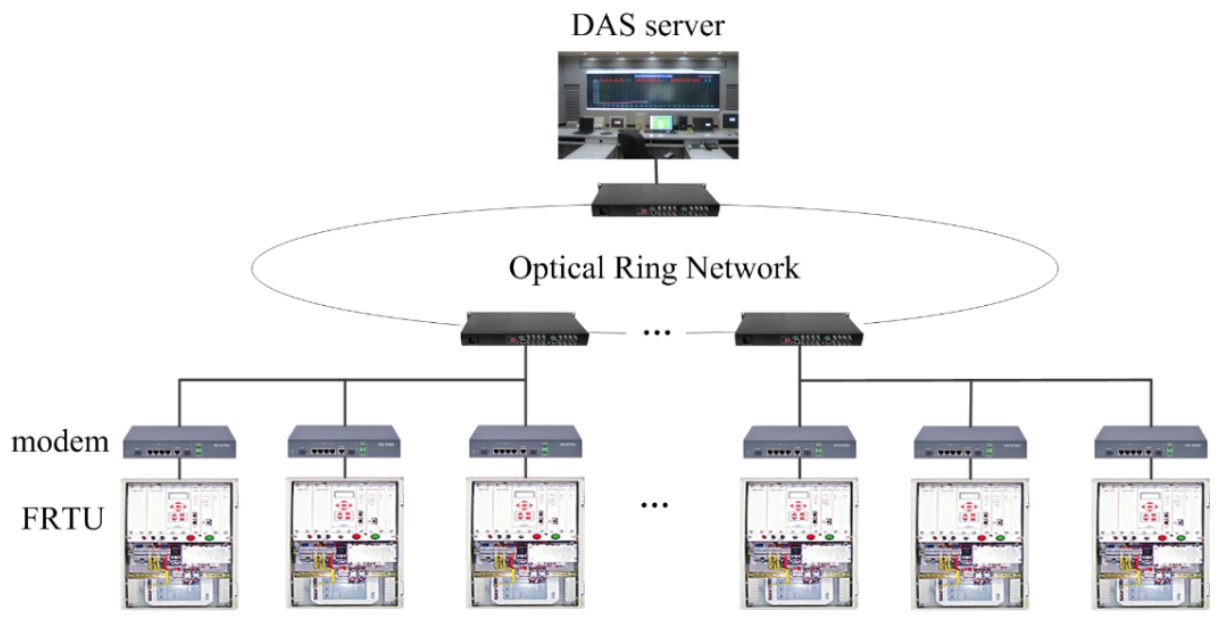

Figure 1. DAS communication architecture.

\subsection{Attack Process}

In order to launch a successful attack, the attackers have to first look for exploitable access points, and then hack other vulnerabilities in the network based on the entry at the access point.

\subsubsection{Selecting Access Point}

In a DAS, potential access points include the servers in a control center as well as the intelligent terminal devices located in remote areas. The terminal devices are usually deployed in remote areas with little physical protection and many of them do not even require a password for authentication [18], while the servers are typically isolated within an electronic security perimeter [12], so it is usually easier to get access to the vulnerabilities in terminal devices than those in control center servers. On the other hand, the exploitation of the vulnerabilities in the servers usually results in severer physical consequences. Thus, the possibility of selecting different vulnerabilities to be access point varies.

\subsubsection{Hacking Other Vulnerabilities}

The second stage of an attack can be modeled as a series of two-person ADGs. The attacker intends to cause the severest physical consequences, while the defender (i.e., the system operators) aims at minimizing this loss. Thus, the game discussed in this paper is basically a non-cooperative ADG. In the ADGs, payoffs for the attacker and the defender are the uppermost elements which are related to the potential physical consequences, vulnerability information and the topology of the distribution system. The Nash equilibrium of the game indicates the attack's attack intention and the optimal defense strategy. The specific analysis method will be presented comprehensively in Section 4.

The overall process of the quantitative vulnerability assessment for a DAS is shown as Figure 2 . The vulnerability adjacency matrix is used to analyze the relationship among different vulnerabilities and will be discussed in Section 5. 


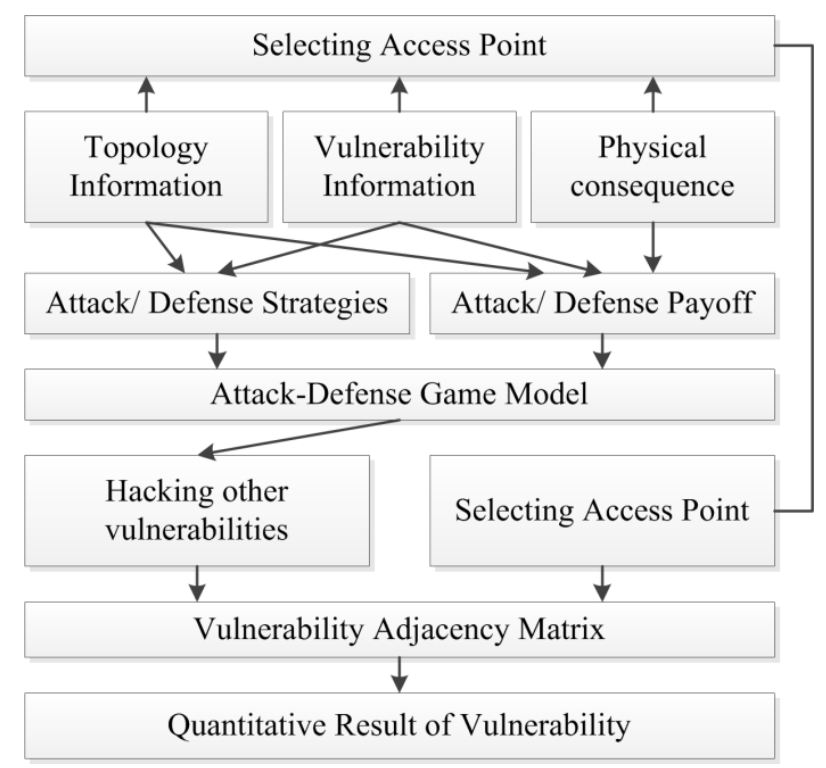

Figure 2. Overall process of vulnerability assessment.

\section{Physical Consequences Analysis}

Based on the discussion in Section 2.1, the physical consequences caused by loss of integrity and availability are analyzed from two levels: terminal devices and control center server. Moreover, both of the normal and fault work condition are considered in this paper.

As shown in Figure 3, a typical multi-sectioned and multi-linked distribution system is given for understanding the potential physical consequences of cyber-attacks. In Figure 3, CB, L, F, S, T represents the circuit breaker, the load, the FRTU, the section switch and the tie switch, respectively. Every switch and circuit breaker is monitored and controlled by control center through an FRTU. The main feeder is divided into several feeder sections by the circuit breaker and other switches.

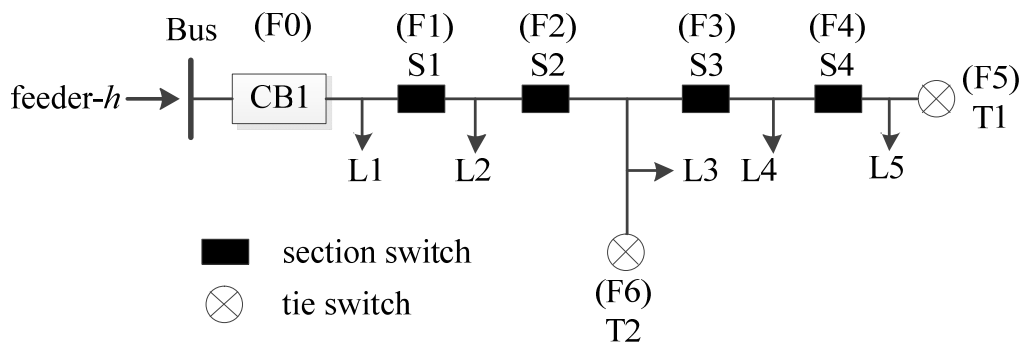

Figure 3. Multi-sectioned and multi-linked network of a distribution system.

When a fault occurs, there are two schemes to implement the restoration function: centralized feeder automation (Scheme 1) and agent-based feeder automation (Scheme 2). As for Scheme 1, all the information will be sent to the control center server from FRTUs, and the server performs fault detection, isolation, and restoration. In Scheme 2, an FRTU can exchange information with its neighbor FRTUs to detect and isolate the fault area. The FRTU of the feeder breaker (i.e., F0 in Figure 3) collects information from other FRTUs in feeder $h$, communicates with the FRTUs of the breakers in its neighbor feeders (i.e., the feeders that are connected with feeder $h$ through tie switches), and then performs restoration function to the power outage area. 


\subsection{Terminal Devices}

\subsubsection{FRTUs of Section Switches}

Control commands and measurement data are the core information being exchanged in a DAS. The control commands are mainly used to operate switches, while the measurement data is used for decision-making. Thus, the consequence caused by loss of integrity in FRTUs of section switches can be determined by:

$$
I_{h, i}=I_{h, i}^{C}+I_{h, i}^{M}
$$

where $I_{h, i}$ represents the consequence caused by loss of integrity in $F_{h, i}$ and $F_{h, i}$ is the $i$ th FRTU in main feeder $h ; I_{h, i}^{C}$ and $I_{h, i}^{M}$ denotes the consequence caused by loss of control command integrity and measurement data integrity in $F_{h, i}$ respectively.

Loss of control commands integrity or measurement data integrity allows an attacker to modify the corresponding information. The modification of control information will result in loss of load directly. For example, a command injection attack on F4 can trip section switch S4 and therefore cause the loss of load L5. With the help of fault detection, isolation and restoration, the power supply to L5 can be restored automatically in several minutes. Thus, the consequence caused by integrity loss of control command is described as:

$$
\begin{gathered}
I_{h, i}^{C}=\sum_{j=i+1}^{N_{h}}\left(\Omega_{h, j} \times t_{h}\right) \\
\Omega_{h, j}=\sum_{k \in S_{h, j}}\left(L_{k} \times \omega_{k}\right)
\end{gathered}
$$

where $\Omega_{h, j}$ is the impact factor of the loads in the $j$ th feeder section of feeder $h$; th is the duration of power outage which is equal to the time needed to apply restoration function in feeder $h ; N_{h}$ is the number of feeder sections in feeder $h ; L_{k}$ and $\omega_{k}$ represents the loading level and the importance of $k$ th load respectively; $S_{h, j}$ is the set of loads in $j$ th feeder section of feeder $h$. The importance of load represents the comprehensive influence on personal and property safety, which is on a scale of 1-5 [26]. The more important the load is, the higher it scores.

The modification of measurement data will mislead the DAS to make an improper control decision. Let us take the measurement data in F2 as an example. When a fault occurs between S1 and S2, S1 experiences a fault current while S2 does not. The correct actions include disconnecting S1 and S2, and closing tie switches to restore power supply to the rest of the distribution system. However, if the measurement data in F2 is tampered, the control center server (in Scheme 1) or agent-based FRTUs (in Scheme 2) would mistakenly believe that S2 experiences a fault current and therefore disconnect S2 and S3. Moreover, CB1 cannot be reclosed as a result that the fault has not been isolated. Thus, L1 and L3 will be wrongly removed compared to the correct control actions. On the other hand, if the fault occurs between S2 and S3, the manipulation of measurement data in F2 may mislead the control center server or agent-based FRTUs into believing that S2 does not experience a fault current. Consequently, $\mathrm{S} 1$ and S2 will be disconnected, and therefore L2 will be left in blackout. Furthermore, because of the fault has not been isolated, fault current appears again in the neighbor feeder (i.e., feeder $h^{\prime}$ ) which 
supplies electricity to L3 after restoration. Therefore, the loads which need to be restored in feeder $h$ and the loads in feeder $h^{\prime}$ will experience outage for some time until the fault is finally isolated by another fault diagnosis process. Based on this discussion, the consequence caused by loss of measurement data integrity can be represented by:

$$
I_{h, i}^{M}=\gamma_{h, i} \times\left(\sum_{j=1}^{i+1}\left(\Omega_{h, j} \times T_{h, j}\right)-\Omega_{h, i} \times T_{h, i}\right)+\gamma_{h, i+1} \times\left(\Omega_{h, i} \times T_{h, i+1}+\left(\sum_{k=i+2}^{N_{h}} \Omega_{h, k}+\sum_{g=1}^{N_{h^{\prime}}} \Omega_{h^{\prime}, g}\right) \times t_{h^{\prime}}\right)
$$

where $\gamma_{h, i}$ is the failure rate of the $i$ th feeder section of feeder $h ; T_{h, i}$ is the time required to repair the permanent failure in $i$ th feeder section of feeder $h ; N_{h^{\prime}}$ is the number of feeder sections in feeder $h^{\prime} ; t_{h^{\prime}}$ is the time needed to apply restore function in feeder $h^{\prime}$. If the fault occurs in the last feeder section, set $t_{h^{\prime}}=0$ because there is no need to close tie switches in this circumstance.

As for availability, it ensures both the control center server and the FRTUs to get the requested information in time. Loss of availability would result in expanding the blackout area. For example, if the trip commands cannot reach S2 when a fault occurs between S1 and S2, S1 and S3 will be disconnected in order to isolate the fault. As a result, L3 will lose electricity additionally.

Therefore, the physical consequence caused by loss of availability in FRTUs of section switches can be denoted as:

$$
A_{h, i}=\gamma_{h, i} \times \Omega_{h, i+1} \times T_{h, i}+\gamma_{h, i+1} \times \Omega_{h, i} \times T_{h, i+1}
$$

where $A_{h, i}$ denotes the consequences caused by loss of availability in $F_{h, i}$.

\subsubsection{FRTUs of Tie Switches}

Tie switches are normally open switches. This enables power wheeling in normal operation and fault restoration. A cyber-attack on the FRTU of a tie switch can result in loss of load. For example, if a fault occurs between S1 and S2, while T1 cannot be closed because of the commands from F5 is modified or not received, just closing T2 may not be able to fully restore the blackout area due to the system constraints. Therefore, the consequence caused by loss of control commands integrity or availability in FTRUs of tie switches can be denoted as:

$$
\begin{aligned}
& I_{h, i}=\sum_{h \in S_{\text {tie }, i}} \sum_{j=1}^{N_{h}-1}\left(\gamma_{h, j} \times T_{h, j} \times \sum_{k \in S_{\text {cut }, h}}\left(L_{k} \times \omega_{k}\right)\right) \quad i \in S_{\text {tie }} \\
& A_{h, i}=\sum_{h \in S_{\text {tie }, i}} \sum_{j=1}^{N_{h}-1}\left(\gamma_{h, j} \times T_{h, j} \times \sum_{k \in S_{\text {cut }, h}}\left(L_{k} \times \omega_{k}\right)\right) \quad i \in S_{\text {tie }}
\end{aligned}
$$

where $S_{t i e}$ is the set of tie switches; $S_{t i e, i}$ is the set of main feeders which can be connected by $i$ th tie switch; $S_{c u t, h}$ is the set of the loads that cannot be restored when a fault occurs in feeder $h$.

In the above equations, the load with a higher importance usually has a higher priority in restoration. Thus, $S_{c u t, h}$ can be confirmed by Algorithm 1 . In this algorithm, $S_{\Delta}$ is the set of loads that need to be restored by closing tie switches; $P_{l}$ is the redundant capacity of other feeders; $\Psi$ is a temporary variable; $N_{S_{\Delta}}$ is the number of the power consumers in $S_{\Delta}$. 


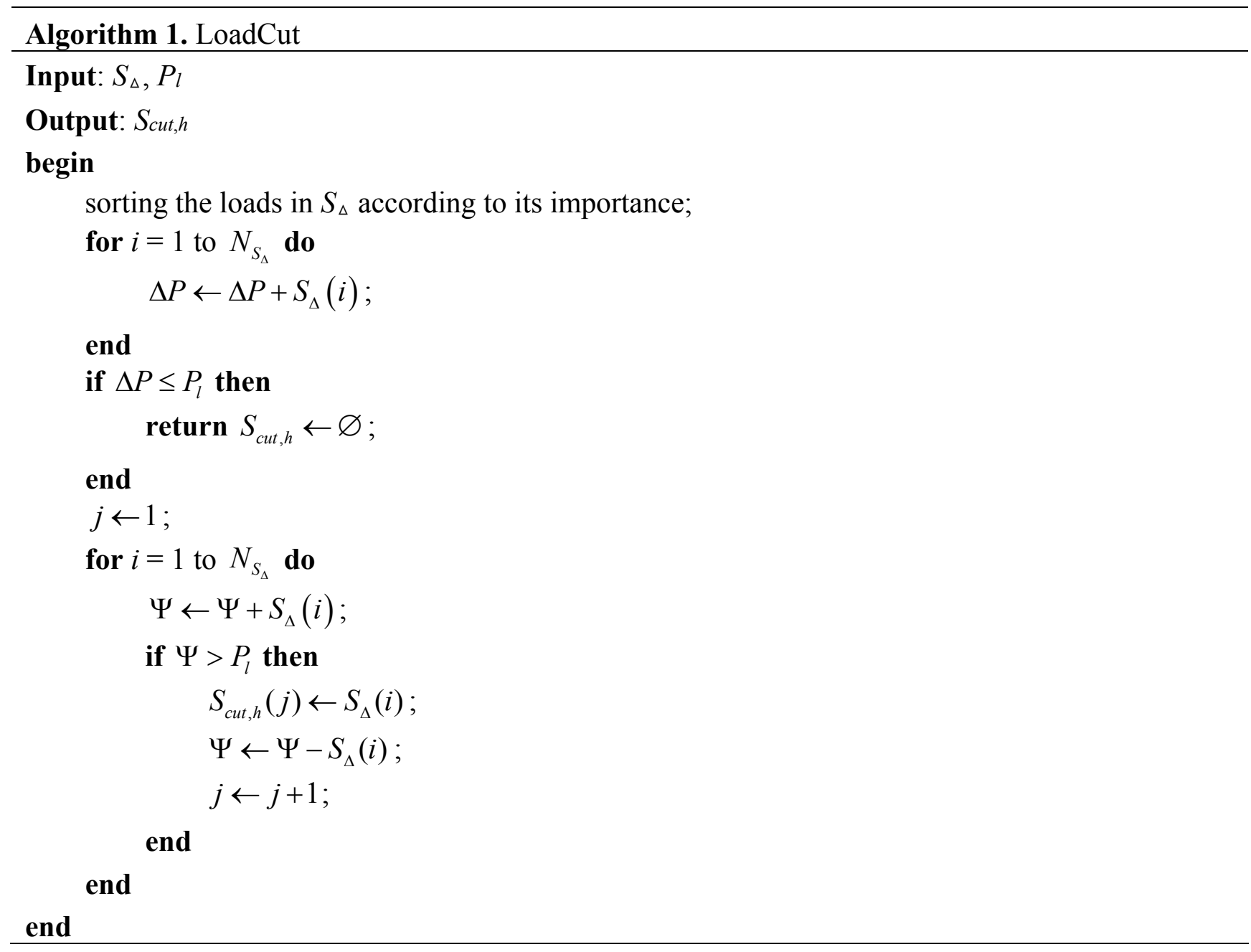

\subsubsection{FRTU of Feeder Breaker}

The FRTU of a feeder breaker plays different roles in different restoration schemes. In Scheme 1, the control center server performs the isolation and restoration strategies. F0 (i.e., the FRTU of feeder breaker in feeder $h$ ) does not communicate with other FRTUs. The consequence caused by loss of integrity in F0 is similar with that of FRTUs of section switches:

$$
I_{h, i}=\sum_{j=1}^{N_{h}}\left(\Omega_{h, j} \times t_{h}\right) \quad i \in S_{h, C B}
$$

where $S_{h, C B}$ is the set of feeder breakers in feeder $h$.

If the close command cannot reach CB1 after fault isolation, the loads in the source side of feeder $h$ will remain in blackout. Thus, the consequence caused by loss of availability in the FRTU of a feeder breaker can be represented as:

$$
A_{h, i}=\sum_{j=2}^{N_{h}}\left(\gamma_{h, j} \times T_{h, j} \times \sum_{k=1}^{j-1} \Omega_{h, k}\right) \quad i \in S_{h, C B}
$$

In Scheme 2, F0 makes the restoration strategy and sends commands to other FRTUs. A modified command from F0 to trip CB1 may lead to blackout in feeder $h$. On the other hand, if a fault occurs in the neighbor feeder of feeder $h, \mathrm{~F} 0$ may send a tampered response to misguide the neighbor feeder into 
believing that feeder $h$ does not have extra power to restore the outage loads. In other words, feeder $h$ is unable to provide restoration power to its neighbor feeders. Therefore, in Scheme 2, the consequence caused by loss of integrity in F0 can be confirmed by:

$$
I_{h, i}=\sum_{j=1}^{N_{h}}\left(\Omega_{h, j} \times t_{h}\right)+\sum_{h^{\prime} \in S_{\text {nei }, h}} \sum_{g=1}^{N_{h^{\prime}}-1}\left(\gamma_{h^{\prime}, g} \times T_{h^{\prime}, g} \times \sum_{k \in S_{\text {cut }, h^{\prime}}}\left(L_{k} \times \omega_{k}\right)\right)
$$

where $S_{n e i, h}$ denotes the set of neighbor feeders of feeder $h$.

If the service of F0 is not available when a fault occurs, the loads will remain in outage as a result of $\mathrm{CB} 1$ and tie switches have not been closed. Furthermore, feeder $h^{\prime}$ cannot get restoration power from feeder $h$ because it cannot get the necessary information from F0. Thus, in Scheme 2, the consequence caused by loss of availability in F0 can be confirmed by:

$$
A_{h, i}=\sum_{j=1}^{N_{h}}\left(\gamma_{h, j} \times T_{h, j} \times \sum_{l=1}^{N_{h}} \Omega_{h, l}\right)+\sum_{h^{\prime} \in S_{\text {nei }, h}} \sum_{g=1}^{N_{h^{\prime}-1}}\left(\gamma_{h^{\prime}, g} \times T_{h^{\prime}, g} \times \sum_{k \in S_{\text {cut }, h^{\prime}}}\left(L_{k} \times \omega_{k}\right)\right)
$$

\subsection{Control Center Server}

The control center server performs supervisory control to the distribution system in normal operation, and plays different roles when a fault occurs according to different schemes.

In Scheme 1, the control center server is in charge of making control decisions and sending control commands to the FRTUs when a fault occurs. If the control center server is attacked, the loss of control commands integrity can result in blackout of the entire distribution system. Thus:

$$
I_{c t r l}=\sum_{h \in S_{F}} \sum_{j=1}^{N_{h}}\left(\Omega_{h, j} \times T_{\text {server }}\right)
$$

Where $I_{c t r l}$ is the consequence caused by loss of information integrity in control center server; $S_{F}$ is the set of feeders; $T_{\text {server }}$ is the time needed to recover the control center server.

If the control center server is not available to formulate control strategies, the fault will not be removed and the loads in the fault feeder will remain in outage. Thus, the consequence caused by loss of information availability in control center server (i.e., $A_{c t r l}$ ) can be represented as:

$$
A_{c t r l}=\sum_{h \in S_{F}} \sum_{i=1}^{N_{h}}\left(\gamma_{h, i} \times T_{h, i} \times \sum_{j=1}^{N_{h}} \Omega_{h, j}\right)
$$

In Scheme 2, the agent-based FRTUs are in charge of fault detection, isolation and restoration. Loss of availability in a control center server will not affect fault isolation and loads restoration. In other words, an attack on the availability of a control center server will not result in loss of load, so $A_{c t r l}=0$. However, the control center server can still trip or close the switches through remote control in normal operations. Thus, the physical consequence caused by loss of integrity in control center server in this scheme is determined by Equation (12). 


\section{DAS Vulnerability Assessment Model}

\subsection{Selecting Access Point}

Both the FRTUs and the control center server in the DAS can be used as initial access points by attackers. The possibility of selecting a specific vulnerability to be access point is mainly related to two factors: (1) the difficulty of getting access to a specific vulnerability; (2) the potential physical consequences of successful exploitation of a vulnerability.

The metrics of access difficulty are shown in Table 1 . The greater the difficulty is, the lower the metric value will be. Table 1 reflects the reality that comprehensive physical protection and network isolation are helpful to prevent a vulnerability from being attacked.

Table 1. Access Difficulty Scoring Evaluation.

\begin{tabular}{cc}
\hline Metric value & Description \\
\hline 0.2 & A vulnerability is of comprehensive physical protection and is local exploitable only. \\
0.5 & A vulnerability is of comprehensive physical protection and is remotely exploitable. \\
0.8 & A vulnerability is of little physical protection and is local exploitable only. \\
1.0 & A vulnerability is of little physical protection and is remotely exploitable. \\
\hline
\end{tabular}

After getting access to the access point, the attackers can launch a further attack by taking advantages of the vulnerabilities in other devices. As a matter of fact, the exploitation of a vulnerability does not always result in the complete loss of integrity and availability. Different vulnerabilities have different impacts on the security properties. For the vulnerability $i$ in device $t$, the potential physical consequence after being attacked takes the following form:

$$
R_{v u l, i}=\left(\boldsymbol{\alpha}_{i} \times \mathbf{C}_{t}^{\mathrm{T}}\right) \times \lambda_{i}
$$

In Equation (14), $R_{v u l, i}$ denotes the potential physical consequence if vulnerability $i$ (i.e., $\left.V_{i}\right)$ is successfully exploited; $\lambda_{i}$ measures the complexity to exploit $V_{i}$, its reference value is provided by the Access Complexity metric in CVSS [19]; $\boldsymbol{C}_{t}=\left[I_{h, t} A_{h, t}\right]$ (if $t$ belongs to terminal devices) or $\boldsymbol{C}_{t}=\left[I_{c t r l} A_{c t r l}\right]$ (if $t$ is a control center server) is a vector of a specific device $t$ which includes two aspects of potential physical consequences; $\boldsymbol{\alpha}_{i}=\left[\alpha_{\text {int }, i} \alpha_{\text {avail, },}\right]$ is a logical array, where $\alpha_{i n t, i}$ and $\alpha_{\text {avail }, i}$ represent whether an attack on $V_{i}$ will result in loss of integrity and availability in device $t$ respectively. If the exploitation of $V_{i}$ does have an impact on the loss of integrity or availability, the corresponding element is assigned to 1 , otherwise the element is assigned to 0 .

Therefore, the possibility of selecting $V_{i}$ to be access point (i.e., $P_{a c c, i}$ ) can be represented as follows:

$$
P_{a c c, i}=\left\{\begin{array}{cc}
\frac{R_{a p, i} \times R_{v u l, i}}{\sum_{j \in S_{a c c}\left(R_{a p, j} \times R_{v u l, j}\right)},} & i \in S_{a c c} \\
0, & i \notin S_{a c c}
\end{array}\right.
$$

where $R_{a p, i}$ and $R_{a p, j}$ represent the difficulty to get access to $V_{i}$ and $V_{j}$ respectively; $S_{a c c}$ is the set of potential access points in a DAS. 


\subsection{Hacking Other Vulnerabilities}

After penetrating an access point, an attacker can get sensitive information about surrounding devices such as device settings and vulnerability information for a next-step attack. As mentioned above, the relationship between the attacker and the system operator can be modeled as a two-person ADG. The attacker's strategy is to select an attack target, and the defender's strategy is to take a defense action. In order to predict the probability distribution of attack actions and defense strategies that reasonable attacker and defender would take, the attacker's payoff and defender's payoff should be analyzed elaborately.

The maximal payoff for an attacker by attacking $V_{j}$ in device $t$ ' can be calculated according to Equation (14). However, the attacker can barely get the maximal payoff because of the defense strategies and access difficulty. Suppose an attack is launched from $V_{i}$, the set of attacker's strategy and defender's strategy can be denoted as $S_{a}^{i}=\left(S_{i, j}^{a}\right)_{1 \times m}$ and $S_{d}^{i}=\left(S_{i, k}^{d}\right)_{1 \times n}$ respectively, where $S_{i, j}^{a}$ represents the strategy of attacking $V_{j}, S_{i, k}^{d}$ represents using $k$ th defense strategy.

The payoff function for an attacker, i.e., $U_{a}\left(S_{i, j}^{a}, S_{i, k}^{d}\right)$, can be represented as follows:

$$
U_{a}\left(S_{i, j}^{a}, S_{i, k}^{d}\right)=\left(R_{v u l, j}-D\left(S_{i, j}^{a}, S_{i, k}^{d}\right)\right) \times R_{a p, j}
$$

where $D\left(S_{i, j}^{a}, S_{i, k}^{d}\right)$ represents the positive impact of the defense strategy $S_{i, k}^{d}$ when the attacker takes the strategy $S_{i, j}^{a}$. The quantitative method of $D\left(S_{i, j}^{a}, S_{i, k}^{d}\right)$ will be presented in the following text.

Because of the real-time requirement of DAS operations and the limited computing power in terminal devices, some common secure methods such as message authentication may have disruptive effects on the normal operation of a DAS [8,27], so both the positive and negative impacts of a specific defense strategy should be taken into consideration in formulating the payoff function for a defender.

The positive impact refers to the defense reward against an attack, i.e., the reduction of attacker's payoff through deploying a defense strategy. Different defense strategies lead to different defense effects. For example, message authentication contributes to checking data integrity. Therefore, the positive impact could be denoted as follows:

$$
D\left(S_{i, j}^{a}, S_{i, k}^{d}\right)=\left(\boldsymbol{\alpha}_{j} \wedge \boldsymbol{\beta}_{k}\right) \times \mathbf{C}_{i^{\prime}}^{\mathbf{T}} \times \lambda_{j}
$$

In Equation (17), $\wedge$ represents the logical AND operation which performs the logical operation on each element of array $\boldsymbol{\alpha}_{j}$ and array $\boldsymbol{\beta}_{k} ; \boldsymbol{\beta}_{k}=\left[\beta_{\text {int }, k} \beta_{\text {avail }, k}\right]$ is a logical array, where $\beta_{\text {int }, k}$ and $\beta_{\text {avail }, k}$ represent whether defense strategy $S_{i, k}^{d}$ contributes to enhance the integrity and availability, respecively. If $S_{i, k}^{d}$ effectively enhances the integrity or availability, the corresponding element is set to be 1 , otherwise it will be 0 .

The negative impacts of a defense strategy mainly include loss of availability of devices and other costs of deploying the defense strategy. In practice, the overall cost of deploying defense strategies in a DAS is usually constrained, so we need to rank the vulnerabilities and then enhance the DAS cyber security in a more effective way, i.e., according to the ranking list. While the cost of deploying the defense strategy for a single device is not a major concern in calculating the defender's payoff. For example, if a defense strategy can significantly increase the cyber security of the DAS, it should be used even if it requires a high cost to deploy. 
In a DAS, both the control center server and the terminal devices require time-critical responses to achieve real-time monitoring and controlling. In other words, only the timely transmitted data is valid. Therefore, considering that the FRTUs in a DAS usually have very limited computing power, some typical defense strategies such as using encryption techniques in message authentication are not always feasible. For example, an over complex cryptographic algorithm will increase the computational complexity and need additional time for encryption and decryption before the information is sent and received. As a result, the real-time transmission of control commands and measurement data requirement might be violated. How to design a cryptographic algorithm which can ensure that the messages can be appropriately encrypted while limiting the latency is a research hotspot $[15,16,28]$, but it is out of the scope of this paper. In this paper, the time needed to transmit information between the control center server and the terminal devices, including transmission time and the extra computation time for encryption and decryption, can be calculated according to the computer network knowledge [29]. If the overall time exceeds a pre-set threshold, the defense strategy is supposed to have a negative impact on the availability of information. The negative impact of $k$ th defense strategy, i.e., $N\left(S_{i, j}^{a}, S_{i, k}^{d}\right)$, is denoted as:

$$
N\left(S_{i, j}^{a}, S_{i, k}^{d}\right)=\lambda_{j} \times \sum_{i \in \Phi_{k}} A_{h, t}
$$

where $\Phi_{k}$ represents the set of devices which are influenced by $k$ th defense strategy.

According to the above discussion, the defender's payoff function can be represented by:

$$
U_{d}\left(S_{i, j}^{a}, S_{i, k}^{d}\right)=D\left(S_{i, j}^{a}, S_{i, k}^{d}\right) \times R_{a p, j}-N\left(S_{i, j}^{a}, S_{i, k}^{d}\right)
$$

Based on the payoff functions for the attack and the defender, i.e., Equation (16) and (19), the probability distribution of attack actions, i.e., $\boldsymbol{\sigma}_{\mathrm{a}}$, can be obtained by solving the Nash equilibrium of the ADG [30]. $\boldsymbol{\sigma}_{\mathbf{a}}=\left(\sigma_{i, j}^{a}\right)_{1 \times m}$, where $\sigma_{i, j}^{a}$ is the possibility of attacking $V_{j}$ from $V_{i}$. If there are multiple equilibrium solutions, the Pareto efficiency criterion is used to identify the final solutions.

\section{Vulnerability Adjacency Matrix}

The quantitative assessment result of a vulnerability denotes the possibility that the vulnerability is attacked. The vulnerability with a higher score is more likely to be attacked. Here, the vulnerability adjacency matrix is introduced to analyze the relationship between different vulnerabilities.

Definition 1: Single-step Vulnerability Adjacency Matrix (SVAM). The element $V_{i, j}$ in an SVAM denotes the possibility that $V_{j}$ is selected to be the next-step attack target when the threat reaches $V_{i}$. The bigger the value of $V_{i, j}$ is, the more likely $V_{j}$ is attacked from $V_{i}$. When $i=1, V_{1, j}$ represents the possibility that vulnerability $j$ is selected to be the access point, thus $V_{i, j}=P_{a c c, j}$; otherwise, the value of $V_{i, j}$ can be determined as:

$$
V_{i, j}= \begin{cases}\sigma_{i, j}^{a}, & j \in S_{i} \\ 0, & j \notin S_{i}\end{cases}
$$

where $S_{i}$ is the set of the vulnerabilities that can be exploited through a single-step attack from $V_{i}$.

SVAM describes the relationship of two vulnerabilities within a single-step attack, but it cannot deal with the multi-step attack scenario. Assuming that there are multiple attack paths between the 
source node $\left(V_{i}\right)$ and the destination node $\left(V_{j}\right)$, the possibilities of selecting different paths can be calculated by Algorithm 2. This algorithm traverses all the nodes based on depth first search strategy, where $E_{i, j}$ is the set of attack paths between $V_{i}$ and $V_{j}, P_{i, j}$ is the set of the possibilities of selecting different paths. Some rings which will result in repetitive computation of the quantitative results may occur in attack paths during traversing. Thus, we use $\pi$ to store the nodes in the attack path, $V_{i} \in \boldsymbol{\pi}$. If the nextstep attack node $V_{n} \in \boldsymbol{\pi}$, the iterative process will be stopped. $P_{\pi}$ is the possibility of selecting the path. In order to get $E_{i, j}$ and $P_{i, j}$, the initial values of $\boldsymbol{\pi}$ and $P_{\pi}$ are $V_{i}$ and 1 , respectively.

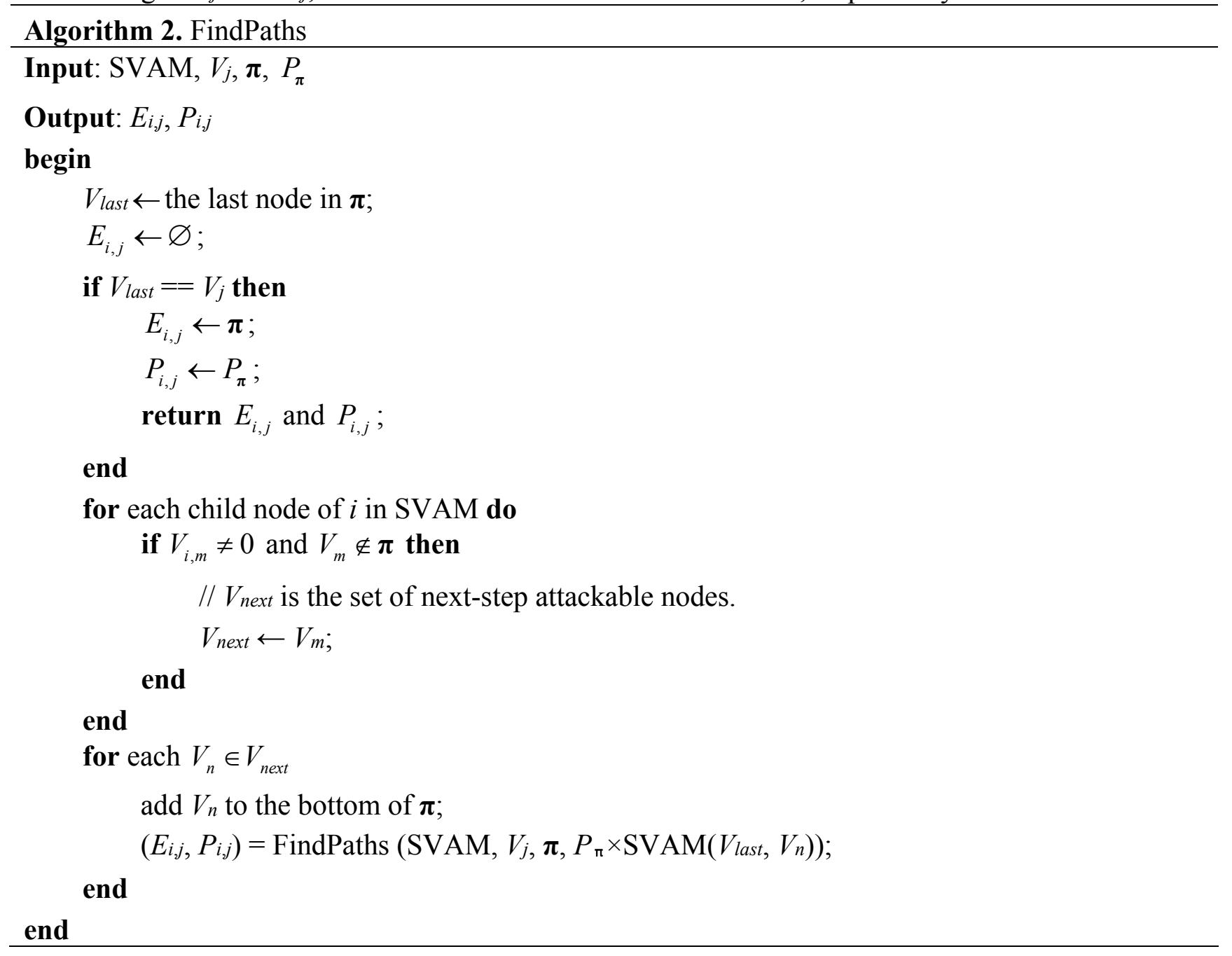

The statistical data of cyber-attacks shows that the length of an attack path is usually shorter than 10 steps. Therefore, the attacks which need more than 10 attack steps should be removed from $E_{i, j}$. In conclusion, the quantitative result of $V_{j}$, i.e., $Q_{j}$, can be denoted as:

$$
Q_{j}=\sum_{k \in E_{1, j}} P_{1, j}^{k}
$$

where $P_{1, j}^{k}$ denotes the possibility of attacking $V_{j}$ through $\mathrm{k} t h$ path.

\section{Case Studies}

The IEEE RBTS bus 2 distribution system [31] is introduced here to illustrate how to apply the proposed assessment model. Both the scenarios of Scheme 1 and Scheme 2 are simulated. Simulation results demonstrated the effectiveness of the method. 
Figure 4 shows the topology of the RBTS bus 2 system and the locations of FRTUs. There are four main feeders, ten section switches and two tie switches in total. Every switch is monitored and controlled by an FRTU. The communication network of the RBTS bus 2 is assumed to be a ring network which is also depicted by the dashed lines in Figure 4.

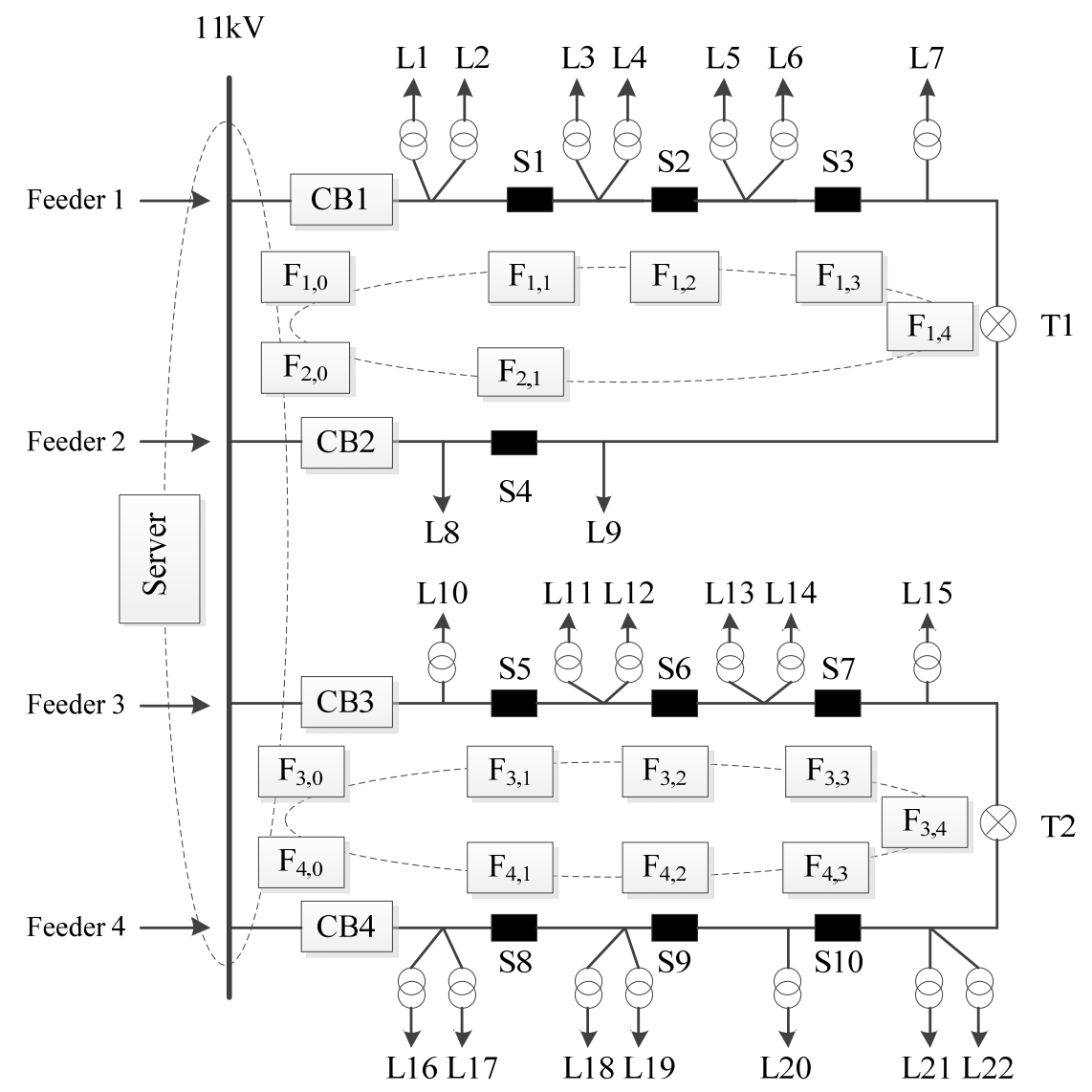

Figure 4. Distribution system for RBTS bus 2 including FRTUs.

In the simulation, the response time to apply restore function is assumed to be $5 \mathrm{~min}$, and the time required to repair a permanent failure or the control center server is assumed to be 60 minutes [3]. The importance of each load is given in Table 2, the other detailed information of RBTS bus 2 is shown in reference [31].

Table 2. The importance of different loads.

\begin{tabular}{ccc}
\hline Load points & Customer type & Load importance \\
\hline $1-3,10-12,17-19$ & residential & 1 \\
8,9 & industrial & 3 \\
$4,5,13,14,20,21$ & government/institution & 3 \\
$6,7,15,16,22$ & commercial & 2 \\
\hline
\end{tabular}

According to the discussion in Section 3, in the scenario of Scheme 1, the potential physical consequences caused by loss of integrity and availability in control center server are $41.751 \mathrm{MWh}$ and 6.7761 MWh, respectively. In Scheme 2, only the attacks on the integrity of control center server would result in loss of load and the result of physical consequence is $41.751 \mathrm{MWh}$. As for the terminal devices, 
the potential physical consequences are shown as Figure 5. As is clearly seen, the physical consequences of attacking FRTUs of feeder circuit breakers become larger in Scheme 2. This is mainly because that an attack on the FRTU of a feeder circuit breaker would affect not only the loads in the related feeder, but also the loads in its neighbor feeder in Scheme 2.

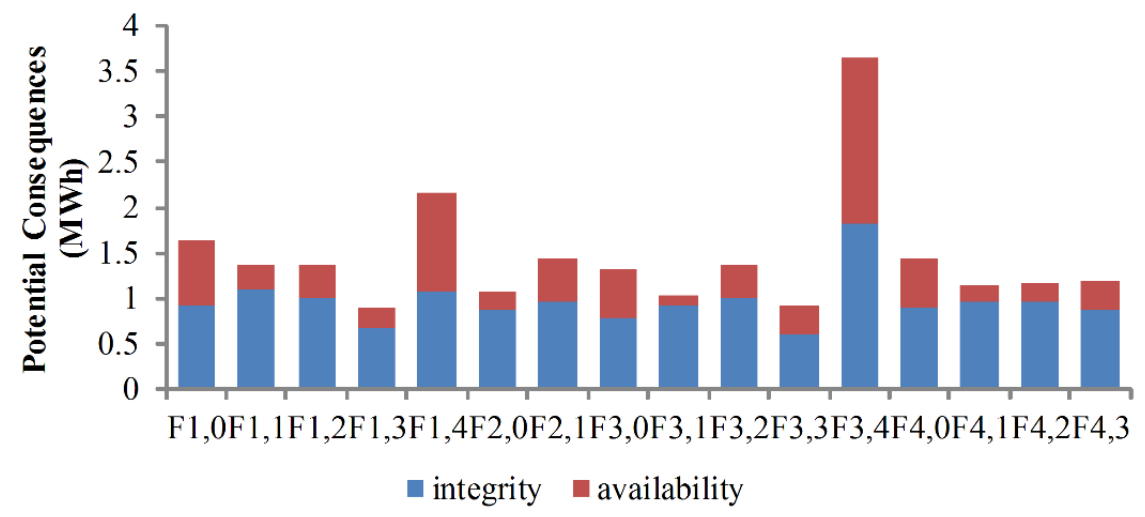

(a)

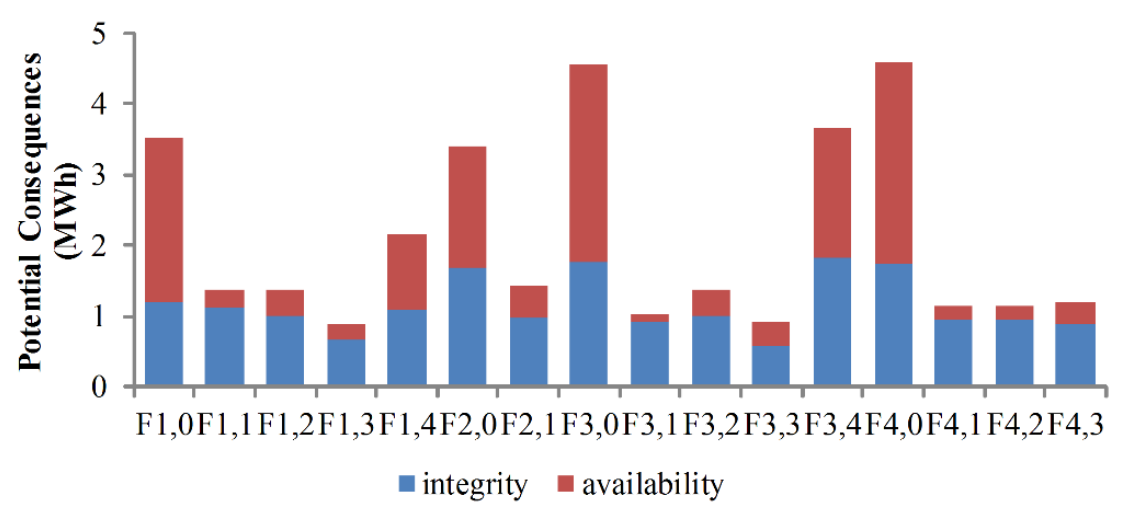

(b)

Figure 5. (a) Physical consequences of attacking FRTUs in Scheme 1; (b) Physical consequences of attacking FRTUs in Scheme 2.

The hypothetical vulnerability information of the DAS in this case study is shown as Table 3, including the types of vulnerabilities and the corresponding access complexity (AC). Among which, "Get administrator rights" and "Privilege escalation" means the exploitation of related vulnerabilities will result in loss of integrity and availability, "Denial of Service" will result in loss of availability, "Unauthorized access" will result in loss of integrity. In practice, the vulnerability information could be acquired by vulnerability scanning.

Taking vulnerability 17 as an example, the maximal payoff for an attacker by attacking this vulnerability in Scheme 1 and Scheme 2 can be calculated as follows:

Scheme 1: $R_{\text {vul }, 17}=(41.75 \times 1+6.78 \times 1) \times 0.71=34.4563 \mathrm{MWh}$;

Scheme $2: R_{v u l, 17}=(41.75 \times 1+0 \times 1) \times 0.71=29.6425 \mathrm{MWh}$.

Thus, by attacking vulnerability 17 , the attacker can get a payoff up to $34.4563 \mathrm{MWh}$ and 29.6425 MWh in Scheme 1 and Scheme 2, respectively. For other vulnerabilities, the maximal payoffs for the attacker are depicted in Figure 6. Obviously, an attacker can cause greater losses by attacking vulnerability 17 than others. 
Table 3. Information of vulnerabilities.

\begin{tabular}{ccccc}
\hline Vul. No. & Affiliated IEDs & Identifier & Type of Vulnerability & AC \\
\hline 1 & $\mathrm{~F}_{1,0}$ & CVE-2011-4034 & Get administrator rights & 0.61 \\
2 & $\mathrm{~F}_{1,1}$ & CVE-2012-0258 & Get administrator rights & 0.61 \\
3 & $\mathrm{~F}_{1,2}$ & CVE-2013-3528 & Get administrator rights & 0.71 \\
4 & $\mathrm{~F}_{1,3}$ & CVE-2012-0258 & Get administrator rights & 0.61 \\
5 & $\mathrm{~F}_{1,4}$ & CVE-2012-3847 & Denial of Service & 0.71 \\
6 & $\mathrm{~F}_{2,0}$ & CVE-2011-4034 & Get administrator rights & 0.61 \\
7 & $\mathrm{~F}_{2,1}$ & CVE-2012-3847 & Denial of Service & 0.71 \\
8 & $\mathrm{~F}_{3,0}$ & CVE-2011-4034 & Get administrator rights & 0.61 \\
9 & $\mathrm{~F}_{3,1}$ & CVE-2011-4056 & Unauthorized access & 0.61 \\
10 & $\mathrm{~F}_{3,2}$ & CVE-2011-4056 & Unauthorized access & 0.61 \\
11 & $\mathrm{~F}_{3,3}$ & CVE-2011-4056 & Unauthorized access & 0.61 \\
12 & $\mathrm{~F}_{3,4}$ & CVE-2012-3847 & Denial of Service & 0.71 \\
13 & $\mathrm{~F}_{4,0}$ & CVE-2011-4034 & Get administrator rights & 0.61 \\
14 & $\mathrm{~F}_{4,1}$ & CVE-2012-0258 & Get administrator rights & 0.61 \\
15 & $\mathrm{~F}_{4,2}$ & CVE-2013-3528 & Get administrator rights & 0.71 \\
16 & $\mathrm{~F}_{4,3}$ & CVE-2012-0258 & Get administrator rights & 0.61 \\
17 & Central Server & CVE-2011-4514 & Privilege escalation & 0.71 \\
18 & Central Server & CVE-2012-3847 & Denial of Service & 0.71 \\
\hline
\end{tabular}

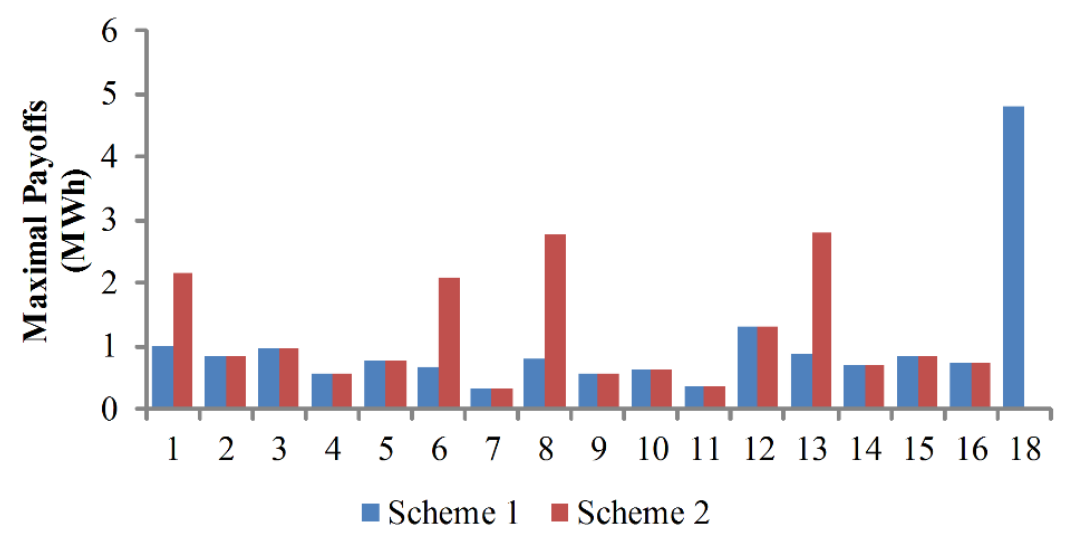

Figure 6. Maximal payoffs of attacking different vulnerabilities.

In a DAS, all the FRTUs and the control center server are potential access points. In general, the control center server is located in the control station and the FRTUs of circuit breakers are located in substations, both of which are well protected in restricted areas. Other FRTUs are located in remote areas with limited physical protection. Therefore, the access difficulty and the possibility that a specific vulnerability is selected to be access point are shown in Table 4. As calculated above, an attacker can cause the severest physical consequence by attacking vulnerability 17 . So, as seen in this table, the possibility of selecting vulnerability 17 to be access point is much higher than others.

After penetrating access points, an attacker can launch further attacks. Some commonly used defense strategies [32] are used in this study. The detailed information of these countermeasures is described in Table 5. Updating patches are helpful to enhance the overall security properties. 
Table 4. Selecting access point.

\begin{tabular}{cccccccc}
\hline Vul. No. & $\boldsymbol{R}_{a p}$ & $\begin{array}{c}\boldsymbol{P}_{a c c} \\
\text { (Scheme 1) }\end{array}$ & $\begin{array}{c}\boldsymbol{P}_{a c c} \\
\text { (Scheme 2) }\end{array}$ & Vul. No. & $\boldsymbol{R}_{a p}$ & $\begin{array}{c}\boldsymbol{P}_{a c c} \\
\text { (Scheme 1) }\end{array}$ & $\begin{array}{c}\boldsymbol{P}_{a c c} \\
\text { (Scheme 2) }\end{array}$ \\
\hline 1 & 0.2 & 0.0118 & 0.0262 & 10 & 1 & 0.0363 & 0.0377 \\
2 & 1 & 0.0489 & 0.0508 & 11 & 1 & 0.0211 & 0.0219 \\
3 & 1 & 0.0574 & 0.0596 & 12 & 1 & 0.0761 & 0.0790 \\
4 & 1 & 0.0321 & 0.0333 & 13 & 1 & 0.0102 & 0.0341 \\
5 & 1 & 0.0451 & 0.0468 & 14 & 0.2 & 0.0408 & 0.0423 \\
6 & 0.2 & 0.0076 & 0.0254 & 15 & 1 & 0.0482 & 0.0500 \\
7 & 1 & 0.0193 & 0.0201 & 16 & 1 & 0.0427 & 0.0443 \\
8 & 1 & 0.0094 & 0.0338 & 17 & 0.2 & 0.4038 & 0.3607 \\
9 & 0.2 & 0.0327 & 0.0340 & 18 & 0.2 & 0.0564 & 0 \\
\hline
\end{tabular}

Table 5. Description of defense strategies.

\begin{tabular}{ccc}
\hline No. & Name & Description \\
\hline 1 & Message authentication & Check the integrity of information \\
2 & Update patch 1 & Patches for Vul. No. 17 \\
3 & Update patch 2 & Patches for Vul. No. 5, 7, 12, and 18 \\
4 & Update patch 3 & Patches for Vul. No. 9, 10, and 11 \\
5 & No countermeasures & None defense measures are deployed \\
\hline
\end{tabular}

The possibility that a specific vulnerability is selected to be the next-step attack node can be calculated by solving the Nash equilibrium of an ADG. For example, in Scheme 2, when the attacker penetrates into vulnerability 1 successfully, the attackable nodes by a single-step attack include vulnerability 2 to vulnerability 6 . According to the analysis in Section 4.2, the Nash equilibrium for attacker is $\boldsymbol{\sigma}_{\mathbf{a}}=$ $(0,0.5211,0,0.4789,0)$, which means that the possibility of choosing vulnerability 3 and vulnerability 5 to be the next-step attack nodes are 0.5211 and 0.4789 respectively.

Based on the analysis of selecting access points and the relationship among different vulnerabilities, we can get the final quantitative results of all vulnerabilities which are shown in Table 6 and Figure 7.

Table 6. Quantitative results of vulnerabilities in different schemes.

\begin{tabular}{cccccc}
\hline Vul. No. & $\begin{array}{c}\text { Score } \\
\text { (Scheme 1) }\end{array}$ & $\begin{array}{c}\text { Score } \\
\text { (Scheme 2) }\end{array}$ & Vul. No. & $\begin{array}{c}\text { Score } \\
\text { (Scheme 1) }\end{array}$ & $\begin{array}{c}\text { Score } \\
\text { (Scheme 2) }\end{array}$ \\
\hline 1 & 0.0118 & 0.2622 & 10 & 0.0363 & 0.0377 \\
2 & 0.0489 & 0.0508 & 11 & 0.0211 & 0.0219 \\
3 & 0.0574 & 0.1652 & 12 & 0.0761 & 0.1928 \\
4 & 0.0321 & 0.0333 & 13 & 0.0102 & 0.3771 \\
5 & 0.0451 & 0.1537 & 14 & 0.0408 & 0.0423 \\
6 & 0.0076 & 0.0455 & 15 & 0.0482 & 0.2978 \\
7 & 0.0193 & 0.0201 & 16 & 0.0427 & 0.0443 \\
8 & 0.0094 & 0.1274 & 17 & 0.4699 & 0.3607 \\
9 & 0.0327 & 0.0340 & 18 & 0.5300 & 0 \\
\hline
\end{tabular}




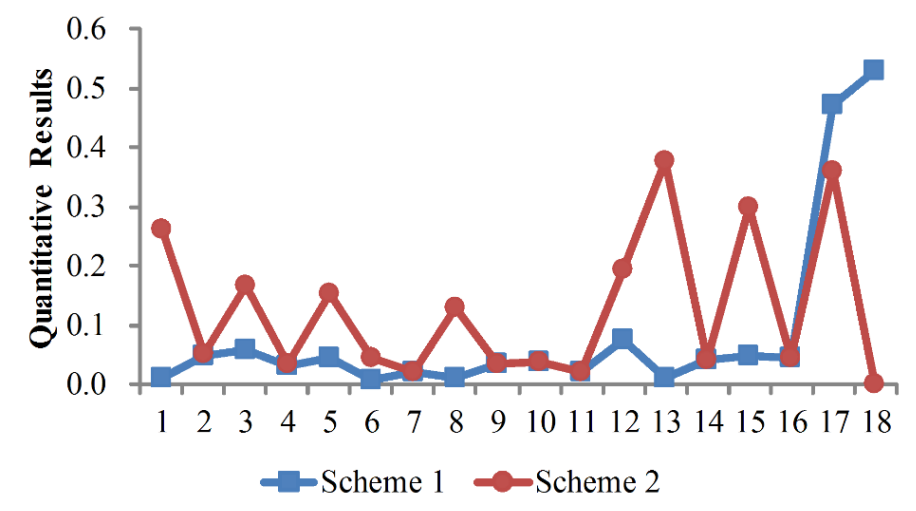

Figure 7. Comparisons of quantitative results of vulnerabilities in different schemes.

As observed from Table 6 and Figure 7, both the vulnerabilities in terminal devices and control center server are likely to be attacked. In Scheme 1, the vulnerabilities in the control center server are more likely to be attacked. This is mainly because that the control center server plays a pivotal role in both normal operation and fault restoration. It collects information from each FRTU, and sends control decisions to them. Although the vulnerabilities in the control center server are harder to exploit, the payoffs are much higher, so the control center server vulnerabilities are more attractive to attackers. Compared to Scheme 1, many FRTUs have a higher possibility to be attacked in Scheme 2. Besides vulnerability 17 which is located in the control center server, vulnerability 1,13 and 15 have relatively higher scores. This is because that the agent-based FRTUs have the ability to exchange information with its neighbor FRTUs, and to restore power to the blackout area when a fault occurs without the help of the control center server. As for vulnerability 18, attacks on it will result in loss of availability, but will not lead to loss of load in Scheme 2 (see Section 3.2), so vulnerability 18 is not attractive to attackers.

The quantitative results denote the possibility that a specific vulnerability will be attacked. Thus, the proposed assessment framework can be used as a tool in distribution system planning and is helpful to identify any cyber security bottlenecks in a distribution system. The vulnerability with a higher score is more likely to be attacked and should receive priority consideration for cyber security.

\section{Conclusions}

Cyber security issues in smart grids merit increasing attention due to the tighter integration of cyber systems with physical power systems. Compared with the control systems in power plants or substations, a DAS is more vulnerable to cyber-attacks. However, ensuring the security of every device in a DAS is both economically inefficient and technically unnecessary.

In this paper, a novel method is proposed for vulnerability assessment and ranking in a DAS. The model includes analyzing the potential physical consequences of cyber-attacks, developing ADG models to simulate the attack processes, and proposing vulnerability adjacency matrix to illustrate the relationship among different vulnerabilities. The case studies based on RBTS bus 2 show the effectiveness and validity of the proposed vulnerability assessment model. 


\section{Acknowledgments}

This work is jointly supported by National Natural Science Foundation of China (No. 51177145, No. 51361130152), and Specialized Research Fund for the Doctoral Program of Higher Education (20120101110112).

\section{Author Contributions}

Xiaming Ye designed the algorithm, performed the simulations and wrote the paper; Junhua Zhao conceived the project; Yan Zhang and Fushuan Wen reviewed and polished the manuscript. All authors discussed the simulation results and approved the assessment methodology.

\section{Conflicts of Interest}

The authors declare no conflict of interest.

\section{References}

1. Ilic, M.D.; Xie, L.; Khan, U.A.; Moura, J.M.F. Modeling of future cyber-physical energy systems for distributed sensing and control. IEEE Trans. Syst. Man Cybern. Part A Syst. Humans 2010, 40, 825-838.

2. Ericsson, G. Cyber security and power system communication-Essential parts of a smart grid infrastructure. IEEE Trans. Power Del. 2010, 25, 1501-1507.

3. Lim, I.H.; Sidhu, T.S.; Choi, M.S.; Lee, S.J.; Hong, S.; Lim, S.I.; Lee, S.W. Design and implementation of multiagent-based distributed restoration system in DAS. IEEE Trans. Power Del. 2013, 28, 585-593.

4. Bou-Harb, E.; Fachkha, C.; Pourzandi, M.; Debbabi, M.; Assi, C. Communication security for smart grid distribution networks. IEEE Commun. Mag. 2013, 51, 42-49.

5. Ericsson, G. Information security for electric power utilities (EPUs)-CIGRE developments on frameworks, risk assessment, and technology. IEEE Trans. Power Del. 2009, 24, 1174-1181.

6. Zio, E.; Sansavini, G. Vulnerability of smart grids with variable generation and consumption: a system of systems perspective. IEEE Trans. Syst. Man Cybern. Syst. 2013, 43, 477-487.

7. National Institute of Standards and Technology, The Smart Grid Interoperability Panel, Cyber Security Working Group. Guidelines for smart grid cyber security. Available online: http://www.nist.gov/smartgrid/upload/nistir-7628_total.pdf (accessed on 10 January 2015).

8. Stouffer, K.; Falco, J.; Scarfone, K. Guide to Industrial Control Systems (ICS) Security; NIST SP 800-82; National Institute of Standards and Technology: Gaithersburg, MD, USA, 2011.

9. Liu, N.; Zhang, J.; Zhang, H.; Liu, W. Security assessment for communication networks of power control systems using attack graph and MCDM. IEEE Trans. Power Del. 2010, 25, 1492-1500.

10. Backhous, S.; Bent, R.; Bono, J.; Lee, R.; Tracey, B.; Wolpert, D.; Xie, D.; Yildiz, Y. Cyber-physical security: A game theory model of human interacting over control systems. IEEE Trans. Smart Grid 2013, 4, 2320-2327.

11. Ten, C.W.; Liu, C.C.; Maninaran, G. Vulnerability assessment of cyber security for SCADA systems. IEEE Trans. Power Syst. 2008, 23, 1836-1846. 
12. Srivastava, A.; Morris, T.; Ernster, T.; Vellaithurai, C.; Pan, S.; Adhikari, U. Modeling cyber-physical vulnerability of the smart grid with incomplete information. IEEE Trans. Smart Grid 2013, 4, 235-244.

13. Zonouz, S., Davis, C.M., Davis, K.R., Berthier, R., Bobab, R.B., Sanders, W.H. SOCCA: A security-oriented cyber-physical contingency analysis in power infrastructures. IEEE Trans. Smart Grid 2014, 5, 3-13.

14. Chen, T.M., Sanchez-Aarnoutse, J.C., Buford, J. Petri net modeling of cyber-physical attacks on smart grid. IEEE Trans. Smart Grid 2011, 2, 741-749.

15. Lim, I.H.; Hong, S.; Chou, M.S.; Lee, S.J.; Kim, T.W.; Lee, S.W. Security protocols against cyber attacks in the distribution automation system. IEEE Trans. Power Del. 2010, 25, 448-455.

16. Sridhar, S.; Hahn, A.; Govindarasu, M. Cyber-physical systems security for the electric power grid. Proc. IEEE 2012, 100, 210-224.

17. Han, G.; Xu, B.; Suonan, J. IEC 61850-based feeder terminal unit modeling and mapping to IEC 60870-5-104. IEEE Trans. Power Del. 2012, 25, 2046-2053.

18. Mo, Y.; Kim, T.H.; Brancik, K.; Dickinson, D; Lee, H.; Perrig, A.; Sinopoli, B. Cyber-physical security of a smart grid infrastructure. Proc. IEEE 2012, 100, 195-209.

19. Mell, P.; Scarfone, K.; Romanosky, S. Common vulnerability scoring system. IEEE Secur. Priv. 2006, 4, 85-89.

20. Information Technology-Security Techniques-Information Security Risk Management; ISO/IEC 27005; International Organization for Standardization/International Electrotechnical Commission (ISO/IEC): Geneva, Switzerland, 2011.

21. Falahati, B.; Fu, Y.; Wu, L. Reliability assessment of smart grid considering direct cyber-power interdependencies. IEEE Trans. Smart Grid 2012, 3, 1515-1524.

22. Ismail, Z.; Leneutre, J.; Bateman, D.; Chen, L. A game theoretical analysis of data confidentiality attacks on smart-grid AMI. IEEE J. Sel. Areas Commun. 2014, 32, 1486-1499.

23. Gamage, T.; Roth, T.; McMillin, B.; Crom, M. Mitigating event based confidentiality violations in smart grids: An information flow security-based approach. IEEE Trans. Smart Grid 2013, 4, 1227-1234.

24. Ko, Y.; Kang, T.; Park, H.; Kim, H.; Nam, H. The FRTU-based fault-zone isolation method in the distribution systems. IEEE Trans. Power Del. 2010, 25, 1001-1009.

25. Zidan, A.; El-Saadany, E. A cooperative multiagent framework for self-healing mechanisms in distribution systems. IEEE Trans. Smart Grid 2012, 3, 1525-1539.

26. Shang, J.; Sheng, X.; Zhang, J.; Zhao, W. The optimized allocation of mobile emergency generator based on the loads importance. In Proceedings of the Asia-Pacific Power and Energy Engineering Conference, New York, NY, USA, 28-31 March 2009; pp. 1-4.

27. Dzung, D.; Naedele, M.; Von Hoff, T.; Crevatin, M. Security for industrial communication systems. Proc. IEEE 2005, 93, 1152-1177.

28. Tsang, P.P.; Smith, S.W. YASIR: A low-latency, high-integrity security retrofit for legacy SCADA systems. In Proceedings of the IFIP TC 11 23rd International Information Security Conference, Milano, Italy, 7-10 September 2008; pp. 445-459.

29. Kurose, J.F., Ross, K.W. Computer Networking: A Top-Down Approach, 5th ed; Addison Wesley: Boston, MA, USA, 2005. 
30. Osborne, M.J. An Introduction to Game Theory; Shanghai University of Finance \& Economics Press: Shanghai, China, 2005.

31. Allan, R.; Billinton, R.; Sjatief, I.; Goel, L.; So, K. A reliability test system for educational purposes-Basic distribution system data and results. IEEE Trans. Power Syst. 1991, 6, 813-820.

32. Jiang, W.; Fang, B.; Zhang, H.; Tian, Z.; Song, X. Optimal network security strengthening using attack-defense game model. In Proceedings of the Sixth International Conference on Information Technology: New Generations, Las Vegas, NV, USA, 27-29 April 2009; pp. 475-480.

(C) 2015 by the authors; licensee MDPI, Basel, Switzerland. This article is an open access article distributed under the terms and conditions of the Creative Commons Attribution license (http://creativecommons.org/licenses/by/4.0/). 